International Journal of Pure and Applied Mathematics

Volume 114 No. 2 2017, 293-299

ISSN: 1311-8080 (printed version); ISSN: 1314-3395 (on-line version)

url: http://www.ijpam.eu

doi: 10.12732/ijpam.v114i2.11

\title{
V-SHAPED TOEPLITZ OPERATOR
}

\author{
Preeti Dharmarha ${ }^{1}$, Savita Rani ${ }^{2} \S$ \\ ${ }^{1}$ Department of Mathematics \\ Hansraj College, University of Delhi \\ Mahatma Hansraj Marg, Delhi, 110007, INDIA \\ ${ }^{2}$ Department of Mathematics \\ Kurukshetra University \\ Kurukshetra, 136119, INDIA
}

\begin{abstract}
A V-shaped Toeplitz operator $V_{\phi}^{\prime}$ is an operator on $L^{2}(\beta)$ defined as $V_{\phi}^{\prime}=\mathrm{W} M_{\phi}^{*}$ , where $M_{\phi}^{*}$ is the weighted multiplication operator and $\mathrm{W}$ is an operator on $L^{2}(\beta)$ given by $\mathrm{W} e_{2 n}=\frac{\beta_{n}}{\beta_{2 n}} e_{n},\left\{e_{n}\right\}_{n \in Z}$ being the orthonormal basis. In this paper we will study its properties.
\end{abstract}

AMS Subject Classification: 47B35

Key Words: V-shaped Toeplitz operator

\section{Introduction}

The Toeplitz operators were introduced in 1911 by O. Toeplitz [5] and many mathematicians followed him with different generalizations of Toeplitz operators. The class of slant Toeplitz operators having the property that the matrices with respect to the standard orthonormal basis could be obtained by eliminating every alternate row of the matrices of the corresponding Toeplitz operators

Received: December 23, 2016

Revised: $\quad$ February 25, 2017

Published: $\quad$ May 6, 2017

(c) 2017 Academic Publications, Ltd. url: www.acadpubl.eu

${ }^{\S}$ Correspondence author 
introduced by $\mathrm{Ho}[4]$ in 1995. These operators are very useful in many applications like prediction theory[2], solution of differential equation[6] and wavelet analysis[3]. However, these studies were made in context of the usual Hardy Spaces and Lorentz spaces. Motivated from these results and the various applications of Toeplitz operators, we introduced and studied V-shaped Toeplitz operators on $L^{2}(\beta)$.We now begin with terminology.

Let $\beta=\left\{\beta_{n}\right\}_{n \in Z}$ be a sequence of positive numbers such that $\beta_{0}=1$, $0<\frac{\beta_{n}}{\beta_{n+1}} \leq 1$, for all $n \geq 0$ and $0<\frac{\beta_{n}}{\beta_{n-1}} \leq 1$ for all $n \leq 0$.

Consider the spaces (see [1],[7])

$$
\begin{gathered}
L^{2}(\beta)=\left\{f(z)=\sum_{n=-\infty}^{\infty} a_{n} z^{n} \mid a_{n} \in C,\right. \\
\left.\|f\|_{\beta}^{2}=\sum_{n=-\infty}^{\infty}\left|a_{n}\right|^{2} \beta_{n}^{2}<\infty\right\},
\end{gathered}
$$

and

$$
\begin{gathered}
H^{2}(\beta)=\left\{f(z)=\sum_{n=0}^{\infty} a_{n} z^{n} \mid a_{n} \in C,\right. \\
\left.\|f\|_{\beta}^{2}=\sum_{n=0}^{\infty}\left|a_{n}\right|^{2} \beta_{n}^{2}<\infty\right\} .
\end{gathered}
$$

Then, $\left(L^{2}(\beta),\|.\|_{\beta}\right)$ is a Hilbert space with an orthonormal basis $\left\{e_{n}(z)=\right.$ $\left.\frac{z^{n}}{\beta_{n}}\right\}_{n \in Z}$ and with respect to the inner product defined by $\left\langle\sum a_{n} z^{n}, \sum b_{n} z^{n}\right\rangle=$ $\sum a_{n} \overline{b_{n}} \beta_{n}^{2}$.

Also, $H^{2}(\beta)$ is a subspace of $L^{2}(\beta)$. If,

$$
L^{\infty}(\beta)=\left\{\phi(z)=\sum_{n=-\infty}^{\infty} a_{n} z^{n} \mid \phi L^{2}(\beta) \subseteq L^{2}(\beta)\right.
$$

and $\exists c \in R$ such that $\|\phi f\|_{\beta} \leq c\|f\|_{\beta}$ for all $\left.f \in L^{2}(\beta)\right\}$, then $L^{\infty}(\beta)$ is a Banach space with a norm defined by

$$
\|\phi\|_{\infty}=\inf \left\{c \mid\|\phi f\|_{\beta} \leq c\|f\|_{\beta}\right.
$$

for all $\left.f \in L^{2}(\beta)\right\}$.

If $\mathrm{P}: L^{2}(\beta) \longrightarrow H^{2}(\beta)$ is the orthogonal projection of $L^{2}(\beta)$ onto $H^{2}(\beta)$, then the weighted Toeplitz operator $T_{\phi}$ on $H^{2}(\beta)[7]$ with symbol $\phi$ in $L^{\infty}(\beta)$ is defined by $T_{\phi} f=P(\phi f)$ for all $f \in H^{2}(\beta)$ 
Let $M_{\phi}^{*}$ denote the weighted multiplication operator on $L^{2}(\beta)$. Then,

$$
M_{\phi}^{*}(f)=\phi f
$$

for all $f \in L^{2}(\beta)$, and

$$
M_{\phi}^{*} e_{k}(z)=\sum_{n=-\infty}^{1} a_{n-k} \frac{\beta_{n} e_{n}(z)}{\beta_{k}}+\sum_{n=2}^{\infty} a_{2-k} \frac{\beta_{n} e_{n}(z)}{\beta_{k}}
$$

for all $k \in Z$.

Definition 1. The V-shaped Toeplitz operator $V_{\phi}^{\prime}$ is an operator on $L^{2}(\beta)$ defined as $V_{\phi}^{\prime}: L^{2}(\beta) \longrightarrow L^{2}(\beta)$ such that $V_{\phi}^{\prime} e_{k}(z)=\sum_{n=-\infty}^{1} \frac{a_{2 n-k}}{\beta_{k}} \beta_{n} e_{n}(z)+$ $\sum_{n=2}^{\infty} \frac{a_{2-k}}{\beta_{k}} \beta_{n} e_{n}(z)$ for $\mathrm{k}=0, \pm 1, \pm 2 \ldots$

The matrix of $V_{\phi}^{\prime}$ is a bilaterally infinite matrix given by

$$
\left(\begin{array}{c|cccc}
\ldots & \ldots & \ldots & \ldots & \ldots \\
\hline \ldots & a_{-2} \frac{\beta_{-1}}{\beta_{0}} & a_{-3} \frac{\beta_{-1}}{\beta_{1}} & a_{-4} \frac{\beta_{-1}}{\beta_{2}} & \ldots \\
\ldots & a_{0} \frac{\beta_{0}}{\beta_{0}} & a_{-1} \frac{\beta_{0}}{\beta_{1}} & a_{-2} \frac{\beta_{0}}{\beta_{2}} & \ldots \\
\ldots & a_{2} \frac{\beta_{1}}{\beta_{0}} & a_{1} \frac{\beta_{1}}{\beta_{1}} & a_{0} \frac{\beta_{1}}{\beta_{2}} & \ldots \\
\ldots & a_{2} \frac{\beta_{2}}{\beta_{0}} & a_{1} \frac{\beta_{2}}{\beta_{1}} & a_{0} \frac{\beta_{2}}{\beta_{2}} & \ldots \\
\ldots & a_{2} \frac{\beta_{3}}{\beta_{0}} & a_{1} \frac{\beta_{3}}{\beta_{1}} & a_{0} \frac{\beta_{3}}{\beta_{2}} & \ldots \\
\ldots & \ldots & \ldots & \ldots & \ldots
\end{array}\right)
$$

Thus we see that this is the matrix obtained by simply eliminating every alternate row of the matrix of weighted multiplication operator $M_{\phi}^{*}$ and multiplying each i-th row by $\frac{\beta_{i}}{\beta_{2 i}}$. If $W: L^{2}(\beta) \longrightarrow L^{2}(\beta)$ is defined as

$$
W e_{2 n}(z)=\frac{\beta_{n}}{\beta_{2 n}} e_{n}(z)
$$

and $W e_{2 n-1}(z)=0$, for all $n \in Z$.

Then the matrix of $\mathrm{W}$ is

$$
\left(\begin{array}{c|cccccc}
\ldots & \ldots & \ldots & \ldots & \ldots & \ldots & \ldots \\
\hline \ldots & \frac{\beta_{0}}{\beta_{0}} & 0 & 0 & 0 & 0 & \ldots \\
\ldots & 0 & 0 & \frac{\beta_{1}}{\beta_{2}} & 0 & 0 & \ldots \\
\ldots & 0 & 0 & 0 & 0 & \frac{\beta_{2}}{\beta_{4}} & \ldots \\
\ldots & \ldots & \ldots & \ldots & \ldots & \ldots & \ldots
\end{array}\right)
$$

and $\|W\|=\sup _{n} \frac{\beta_{n}}{\beta_{2 n}} \leq 1$. 
Below, we will prove the following result.

Theorem 2. (i) $V_{\phi}^{\prime}(f)=W M_{\phi}^{*} f$ for all $f \in L^{2}(\beta)$.

(ii) $W=V_{1}^{\prime}$.

Proof. (i) We have

$$
\begin{aligned}
W M_{\phi}^{*} e_{k}(z) & =W \sum_{n=-\infty}^{1} a_{n-k} \frac{\beta_{n} e_{n}(z)}{\beta_{k}}+\sum_{n=2}^{\infty} a_{2-k} \frac{\beta_{n} e_{n}(z)}{\beta_{k}} \\
& =\sum_{n=-\infty}^{1} \frac{a_{2 n-k}}{\beta_{k}} \beta_{n} e_{n}(z)+\sum_{n=2}^{\infty} \frac{a_{2-k}}{\beta_{k}} \beta_{n} e_{n}(z) \\
& =V_{\phi}^{\prime} e_{k}(z) .
\end{aligned}
$$

(ii) Take $\phi(z)=1$ in the above result, then

$$
V_{1}^{\prime}=W M_{1}^{*}=W
$$

Hence we can now give an alternate definition of V-Shaped Toeplitz operator $V_{\phi}^{\prime}$ as $V_{\phi}^{\prime}(f)=W M_{\phi}^{*} f=\mathrm{W}(\phi f)$

Theorem 3. $V_{\phi}^{\prime}$ is bounded.

Proof. We have

$$
V_{\phi}^{\prime}=W M_{\phi}^{*}
$$

Therefore

$$
\begin{aligned}
\left\|V_{\phi}^{\prime}\right\| & =\left\|W M_{\phi}^{*}\right\| \\
& \leq\|W\|\left\|M_{\phi}^{*}\right\| \\
& \leq\|\phi\|_{\infty} .
\end{aligned}
$$

Since $\left\|M_{\phi}^{*}\right\|=\|\phi\|_{\infty}$ as shown by Shields in [1].

\section{Algebraic Properties}

In this section we will give algebraic properties of V-shaped Toeplitz operator.

Property 1. Determinant of the matrix of $V$-shaped Toeplitz operator $V_{\phi}^{\prime}$ is zero. 
Proof. As the matrix of $V_{\phi}^{\prime}$ is

$$
\left(\begin{array}{c|cccc}
\ldots & \ldots & \ldots & \ldots & \ldots \\
\hline \ldots & a_{-2} \frac{\beta_{-1}}{\beta_{0}} & a_{-3} \frac{\beta_{-1}}{\beta_{1}} & a_{-4} \frac{\beta_{-1}}{\beta_{2}} & \ldots \\
\ldots & a_{0} \frac{\beta_{0}}{\beta_{0}} & a_{-1} \frac{\beta_{0}}{\beta_{1}} & a_{-2} \frac{\beta_{0}}{\beta_{2}} & \ldots \\
\ldots & a_{2} \frac{\beta_{1}}{\beta_{0}} & a_{1} \frac{\beta_{1}}{\beta_{1}} & a_{0} \frac{\beta_{1}}{\beta_{2}} & \ldots \\
\ldots & a_{2} \frac{\beta_{2}}{\beta_{0}} & a_{1} \frac{\beta_{2}}{\beta_{1}} & a_{0} \frac{\beta_{2}}{\beta_{2}} & \ldots \\
\ldots & \ldots & \ldots & \ldots & \ldots
\end{array}\right)
$$

From the above matrix we observe that $a_{i}$ 's in each column are same after some values of k's. Now to verify that determinant is zero we will use the principle of mathematical induction to prove the result for $n \geq 3$. First of all we will prove the result for $3 \times 3$ and $4 \times 4$ matrix.

For $3 \times 3$ matrix

$$
\begin{aligned}
& \quad\left|\begin{array}{ccc}
a_{0} \frac{\beta_{0}}{\beta_{0}} & a_{-1} \frac{\beta_{0}}{\beta_{1}} & a_{-2} \frac{\beta_{0}}{\beta_{2}} \\
a_{2} \frac{\beta_{1}}{\beta_{0}} & a_{1} \frac{\beta_{1}}{\beta_{1}} & a_{0} \frac{\beta_{1}}{\beta_{2}} \\
a_{2} \frac{\beta_{2}}{\beta_{0}} & a_{1} \frac{\beta_{2}}{\beta_{1}} & a_{0} \frac{\beta_{2}}{\beta_{2}}
\end{array}\right| \\
& =a_{0} \frac{\beta_{0}}{\beta_{0}}\left(a_{0} a_{1}-a_{0} a_{1}\right)-a_{-1} \frac{\beta_{0}}{\beta_{1}}\left(a_{0} a_{2} \frac{\beta_{1}}{\beta_{0}}-a_{0} a_{2} \frac{\beta_{1}}{\beta_{0}}\right)+a_{-2} \frac{\beta_{0}}{\beta_{2}}\left(a_{1} a_{2} \frac{\beta_{2}}{\beta_{0}}-a_{1} a_{2} \frac{\beta_{2}}{\beta_{0}}\right) \\
& \quad=0 .
\end{aligned}
$$

Hence the determinant of $3 \times 3 \mathrm{~V}$-shaped Toeplitz matrix is zero.

Now we will prove the result for $4 \mathrm{x} 4$ matrix.

$$
\begin{array}{rrrr}
a_{-2} \frac{\beta_{-1}}{\beta_{0}} & a_{-3} \frac{\beta_{-1}}{\beta_{1}} & a_{-4} \frac{\beta_{-1}}{\beta_{2}} & a_{-5} \frac{\beta_{-1}}{\beta_{3}} \\
a_{0} \frac{\beta_{0}}{\beta_{0}} & a_{-1} \frac{\beta_{0}}{\beta_{1}} & a_{-2} \frac{\beta_{0}}{\beta_{2}} & a_{-3} \frac{\beta_{0}}{\beta_{3}} \\
a_{2} \frac{\beta_{1}}{\beta_{0}} & a_{1} \frac{\beta_{1}}{\beta_{1}} & a_{0} \frac{\beta_{1}}{\beta_{2}} & a_{-1} \frac{\beta_{1}}{\beta_{3}} \\
a_{2} \frac{\beta_{2}}{\beta_{0}} & a_{1} \frac{\beta_{2}}{\beta_{1}} & a_{0} \frac{\beta_{2}}{\beta_{2}} & a_{-1} \frac{\beta_{2}}{\beta_{3}}
\end{array}\left|=a_{-2} \frac{\beta_{-1}}{\beta_{0}}\right| \begin{array}{ccc}
a_{-1} \frac{\beta_{0}}{\beta_{1}} & a_{-2} \frac{\beta_{0}}{\beta_{2}} & a_{-3} \frac{\beta_{0}}{\beta_{3}} \\
a_{1} \frac{\beta_{1}}{\beta_{1}} & a_{0} \frac{\beta_{1}}{\beta_{2}} & a_{-1} \frac{\beta_{1}}{\beta_{3}} \\
a_{1} \frac{\beta_{2}}{\beta_{1}} & a_{0} \frac{\beta_{2}}{\beta_{2}} & a_{-1} \frac{\beta_{2}}{\beta_{3}}
\end{array} \mid
$$




$$
=0 \text {. }
$$

As we see above that if in $3 \times 3$ determinant $a_{i}^{\prime} s$ are same in two rows then the determinant is zero. Hence the determinant of $4 \mathrm{x} 4$ matrix is zero. By using induction hypothesis we can prove easily that if the determinant of mxm matrix is zero then the determinant of $(\mathrm{m}+1) \mathrm{x}(\mathrm{m}+1)$ matrix is zero. Hence the result is true for all $n \geq 3$.

From this we can generalize that the determinant of bilateraly infinite matrix of V-shaped Toeplitz operator is zero.

Remark 2. As we have seen above that the determinant of bilateraly infinite matrix of $\mathrm{V}$-shaped Toeplitz matrix is zero but to obtain shape $\mathrm{V}$ the order of matrix must be odd.

Property 3. $V_{\phi}^{\prime}$ does not commute with $M_{z}^{*}$.

Proof.

$$
\begin{aligned}
M_{z}^{*} V_{\phi}^{\prime} e_{k}(z) & =M_{z}^{*}\left(\sum_{n=-\infty}^{1} \frac{a_{2 n-k}}{\beta_{k}} \beta_{n} e_{n}(z)+\sum_{n=2}^{\infty} \frac{a_{2-k}}{\beta_{k}} \beta_{n} e_{n}(z)\right) \\
& =z\left(\sum_{n=-\infty}^{1} \frac{a_{2 n-k}}{\beta_{k}} \beta_{n} \frac{z^{n}}{\beta_{n}}+\sum_{n=2}^{\infty} \frac{a_{2-k}}{\beta_{k}} \beta_{n} \frac{z^{n}}{\beta_{n}}\right) \\
& =\sum_{n=-\infty}^{1} \frac{a_{2 n-k}}{\beta_{k}} z^{n+1}+\sum_{n=2}^{\infty} \frac{a_{2-k}}{\beta_{k}} z^{n+1} \\
& \left.=\sum_{n=-\infty}^{1} \frac{a_{2 n-k}}{\beta_{k}} \beta_{n+1} e_{n+1}(z)+\sum_{n=2}^{\infty} \frac{a_{2-k}}{\beta_{k}} \beta_{n+1} e_{n+1}(z)\right) \ldots(1) \\
V_{\phi}^{\prime} M_{z}^{*} e_{k}(z)= & V_{\phi}^{\prime} z e_{k}(z) \\
= & V_{\phi}^{\prime} \frac{\beta_{k+1}}{\beta_{k}} e_{k+1}(z) \\
= & \frac{\beta_{k+1}}{\beta_{k}} V_{\phi}^{\prime}\left(e_{k+1}(z)\right) \\
= & \frac{\beta_{k+1}}{\beta_{k}}\left(\sum_{n=-\infty}^{1} \frac{a_{2 n-k+1}}{\beta_{k+1}} \beta_{n} e_{n}(z)+\sum_{n=2}^{\infty} \frac{a_{1-k}}{\beta_{k+1}} \beta_{n} e_{n}(z)\right) \\
& \left.\sum_{n=-\infty} \frac{a_{2 n-k+1}}{\beta_{k}} \beta_{n} e_{n}(z)+\sum_{n=2}^{\infty} \frac{a_{1-k}}{\beta_{k}} \beta_{n} e_{n}(z)\right) \ldots(2)
\end{aligned}
$$


From (1) and (2), we see that $M_{z}^{*} V_{\phi}^{\prime} \neq V_{\phi}^{\prime} M_{z}^{*}$

Property 4. The mapping $\phi \longrightarrow V_{\phi}^{\prime}$ is linear.

Proof. $V_{(\alpha \phi+\beta \psi)}^{\prime}=W M_{(\alpha \phi+\beta \psi)}^{*}=\alpha W M_{\phi}^{*}+\beta W M_{\psi}^{*}=\alpha V_{\phi}^{\prime}+\beta V_{\psi}^{\prime}$.

Hence the mapping is linear.

\section{References}

[1] A. L. Shields, Weighted Shift Operators and Analytic Function Theory. Topics in Operator Theory, Math. Surveys 13, Amer. Math. Soc. Providence, R. I., 1974.

[2] Helson and Szego, A Problem in Prediction Theory,Ann. Math. Pura Appl.,51, No.1(1960), 107-138, doi: 10.1007/BF02410947.

[3] L. Villemoes, Wavelet Analysis of Refinement Equations.SIAM J. Math. Analysis, 25, No.5(1994), 1433-1460.

[4] M. C. Ho., Properties of Slant Toeplitz Operators.Indiana Univ. Math. J.,45, No.3(1996), 843-862, doi: 10.1512/iumj.1996.45.1973.

[5] O. Toeplitz, Zur Theorie Der Quadratishen and Bilinearan Formen Von Unendlichvielen Veranderlichen.Math. Ann70(1911), 351-376.

[6] T. Goodman, C. Micchelli and J.Ward, Spectral Radius Formula for Subdivision Operators, Recent Advances in Wavelet Analysis, ed. L. Schumaker and G. Webb., Academic Press(1994), 335-360.

[7] V. Lauric, On a weighted Toeplitz operator and its commutant. Int.J. Math. and Math.Sci.,2005, No.6(2005),823-835, doi: 10.1155/IJMMS.2005.823. 
